ISSN 1112-9867

Available online at

http://www.jfas.info

\title{
ELABORATION AND DIELECTRIC CHARACTERIZATION OF A DOPED FERROELECTRIC MATERIAL TYPE PZT
}

\author{
M. Abba*, Z. Necira, N. Abdessalem, A. Meklid, H. Menasra, A. Boutarfaia
}

Département de chimie, laboratoire de chimie appliquée, université de Biskra, Algérie.

Received: 28 August 2013 / Accepted: 17 December 2013 / Published online: 30 June 2013

\begin{abstract}
The main objective of this work is based on the synthesis and dielectric characterization of a new material in ceramic PZT with a perovskite structure $\mathrm{ABO}_{3}$. We are interested to study the Quaternary system (doping in site A and site B) of general formula: $\mathrm{Pb}_{0.96} \mathrm{Ba}_{0.02} \mathrm{Ca}_{0.02}\left[\left(\mathrm{Zr}_{0.52} \mathrm{Ti}_{0.48}\right)_{0.94}\left(\mathrm{Zn}_{1 / 3} \mathrm{Ta}_{2 / 3}\right)_{0.03}\left(\mathrm{In}_{1 / 3} \mathrm{Sb}_{2 / 3}\right)_{0.03}\right] \mathrm{O}_{3}$ short PZT-PBC-ZTIS. The sample selected for this study was prepared by the method of synthesis with solid way. Heat treatment was applied to these compositions at different temperatures: 1100, 1150,1180 and $1200{ }^{\circ} \mathrm{C}$ successively to optimize the sintering temperature optimal where the density of the sample is maximum (near theoretical density) and therefore the product has better physical quality. The study of dielectric properties of all samples showed a high permittivity dielectric $\varepsilon \mathrm{r}=18018$, low dielectric loss: $\operatorname{tg} \delta=7.62 \%$, for the composition sintered to $1180^{\circ} \mathrm{C}$ included in the phase morphotropique zone (FMP).
\end{abstract}

Key words: Ceramics PZT, Dielectric properties, Dielectric constant / loss constant.

\section{INTRODUCTION}

Les composées à base d'oxyde de formule générale $\mathbf{P b}\left(\mathbf{Z r}_{\mathbf{x}} \mathbf{T i}_{1-\mathbf{x}}\right) \mathbf{O}_{\mathbf{3}}$ de structure pérovskite $\left(\mathrm{ABO}_{3}\right)$ appelées PZT présentent des propriétés particulièrement intéressantes. Elles sont ferroélectriques dans une grande gamme de températures y compris à température ambiante [1-4].

Author Correspondence, e-mail: abbamalika@gmail.com ICID: 1078189 
En fait, ce sont des matériaux qui se prêtent bien aux investigations expérimentales et qui entrent dans la fabrication industrielle de nombreux composants électroniques comme dispositifs à haute fréquence, résonateurs, transducteurs électromécaniques, capteurs, condensateurs électriques, moteurs piézoélectriques etc...Ceci est grâce à leurs propriétés diélectrique très intéressantes et à leurs couplage électromécanique [5,6].

Les chercheurs ont mis en évidence l'intense activité piézoélectrique de ces matériaux surtout aux compositions proches de la frontière morphotropique de phase noté FMP, où coexistent les deux phases tétragonale et rhomboédrique. Ces compositions (proches de la FMP) exhibent les meilleurs caractéristiques électriques (diélectriques, piézoélectriques ferroélectriques, etc...)

Les propriétés de ces matériaux dépendent de leur microstructure qui, elle-même, est entièrement déterminée par la composition chimique et par le cycle de leur élaboration. Ces propriétés sont généralement améliorées par l'ajout de plusieurs cations qui vont se substituer au $\mathrm{Pb}^{+2}$ en site (A) et/ou au couple $\left(\mathrm{Zr}^{+4} / \mathrm{Ti}^{+4}\right)$ en site (B) de la pérovskite.

Dans le cadre de notre travail, nous nous sommes intéressés à la synthèse de la solution solide $\mathrm{Pb}_{0.96} \mathrm{Ba}_{0.02} \mathrm{Ca}_{0.02}\left[\left(\mathrm{Zr}_{y} \mathrm{Ti}_{1-y}\right)_{0.94}\left(\mathrm{Zn}_{1 / 3} \mathrm{Ta}_{2 / 3}\right)_{0.03}\left(\mathrm{In}_{1 / 3} \mathrm{Sb}_{2 / 3}\right)_{0.03} \mathrm{O}_{3}\right.$ et voir l'effet du frittage sur la microstructure et les propriétés diélectriques.

\section{METHODES EXPERIMENTALES}

Les compositions étudiées ont été synthétisées par voie chimique à l'état solide. Des matières premières ont été mélangées et broyées pendant six heures suivi d'une calcination préliminaire à $900^{\circ} \mathrm{C}$ pendant 2 heures avec une vitesse de chauffage de $2^{\circ} \mathrm{C} / \mathrm{min}$. Le mélange calciné est rebroyé pendant six heures puis pastillés sous $3500 \mathrm{Kg} / \mathrm{cm}^{2}$. Afin d'étudier l'influence de la température de frittage sur les propriétés diélectriques, de la céramique PZT-BCZTIS, les pastilles sont frittés à quatre températures différentes, $1100,1150,1180$, et $1200{ }^{\circ} \mathrm{C}$ en prenant compte du profil thermique de frittage; cinétique : $2 \% \mathrm{~min}$, palier : $2 \mathrm{~h}$ et de l'atmosphère de frittage, qui a été choisi toujours sur la base des résultats obtenus antérieurement [7-9]. Les spectres de nos poudres calcinées et frittées à différentes températures sont réalisés à température ambiante avec $2 \theta$ entre $10^{\circ}$ et $90^{\circ}$ sur un diffractomètre vertical, "BRUKER-AXE type D8" au sein de laboratoire des Rayons X de l'université de Biskra. Ce dernier travaille en utilisant les radiations $\mathrm{K}_{\alpha} \mathrm{du}$ cuivre, et il fonctionne sous une haute tension de $40 \mathrm{KV}$ et un courant de $40 \mathrm{~mA}$. Le logiciel utilisé pour la caractérisation des différentes raies est l'X'PertHighScore.

Les pastilles sont métallisées à l'aide d'une couche mince de la pâte d'argent sur les deux surfaces de la pastille. Après séchage, les pastilles métallisées ont subi une calcination à $750^{\circ} \mathrm{C}$ pendant 
trente minutes. Les variations de la constante diélectrique, la résistivité et l'angle des pertes ont été examinée en utilisant un pont de mesure de type RLC.

\section{RESULTATS ET DISCUSSION}

La figure 1, illustre le diagramme de diffraction de cette poudre de PZT-BCZTIS calciné à $900^{\circ} \mathrm{C}$, dans le domaine angulaire $10^{\circ}<2 \theta<90^{\circ}$ qui semble être suffisant pour l'identification de la phase formée. D'après ce diagramme on remarque l'existence d'une phase unique de type pérovskite qui est caractéristique de la phase PZT [10], ainsi la phase cherchée est totalement formée (pas de phase secondaire) à $900^{\circ} \mathrm{C}$.

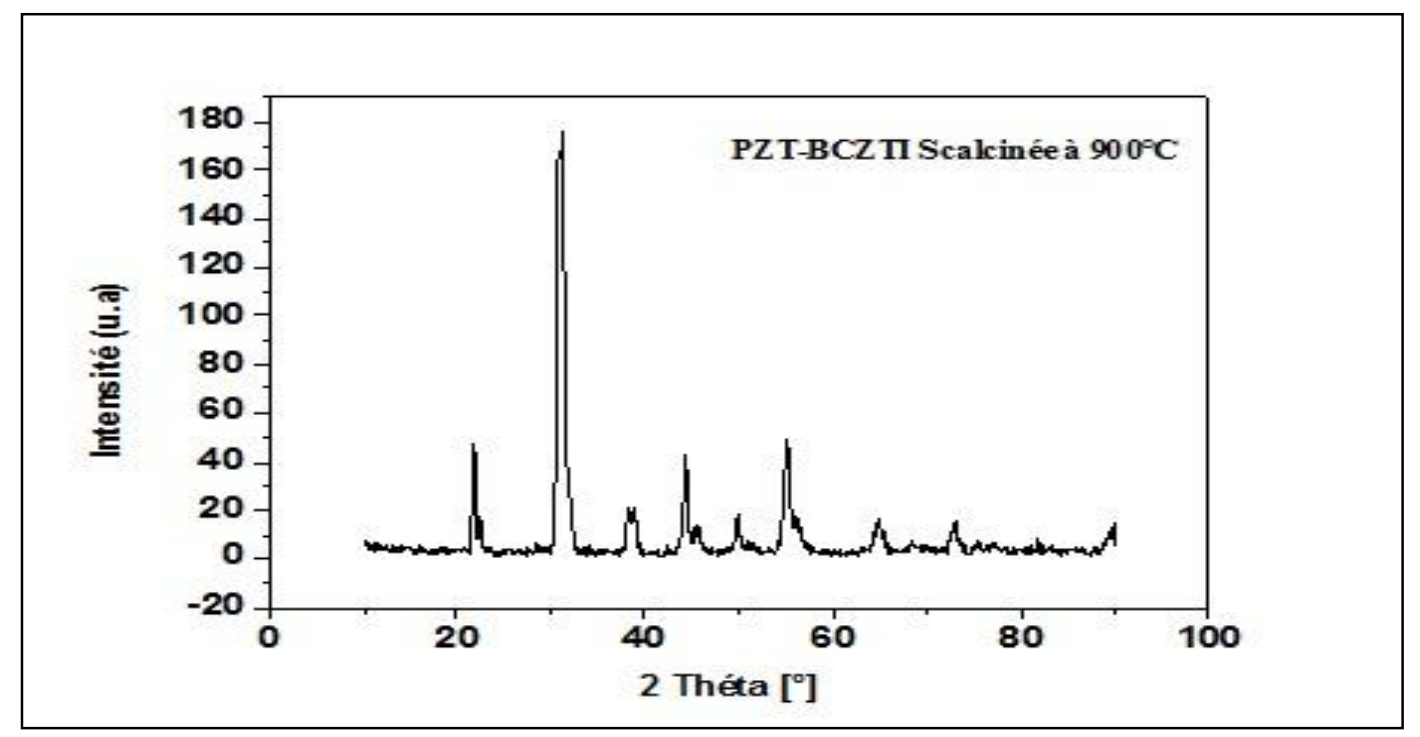

Fig.1. Diagramme de DRX de la PZT-BCZTIS calcinée à $900^{\circ} \mathrm{C}$.

Dans un second temps, et pour voir l'influence de la température de frittage sur la stabilité de la phase formée, les diffractogrammes de la poudre de PZT-BCZTIS frittée à différentes températures sont enregistrés dans le même domaine angulaire $10^{\circ}<2 \theta<90^{\circ}$.La figure .2 , illustre les différents diagrammes des échantillons frittés à ; 1100, 1150, 1180 et $1200{ }^{\circ} \mathrm{C}$ respectivement. 


\section{PZT-BCZTIS}
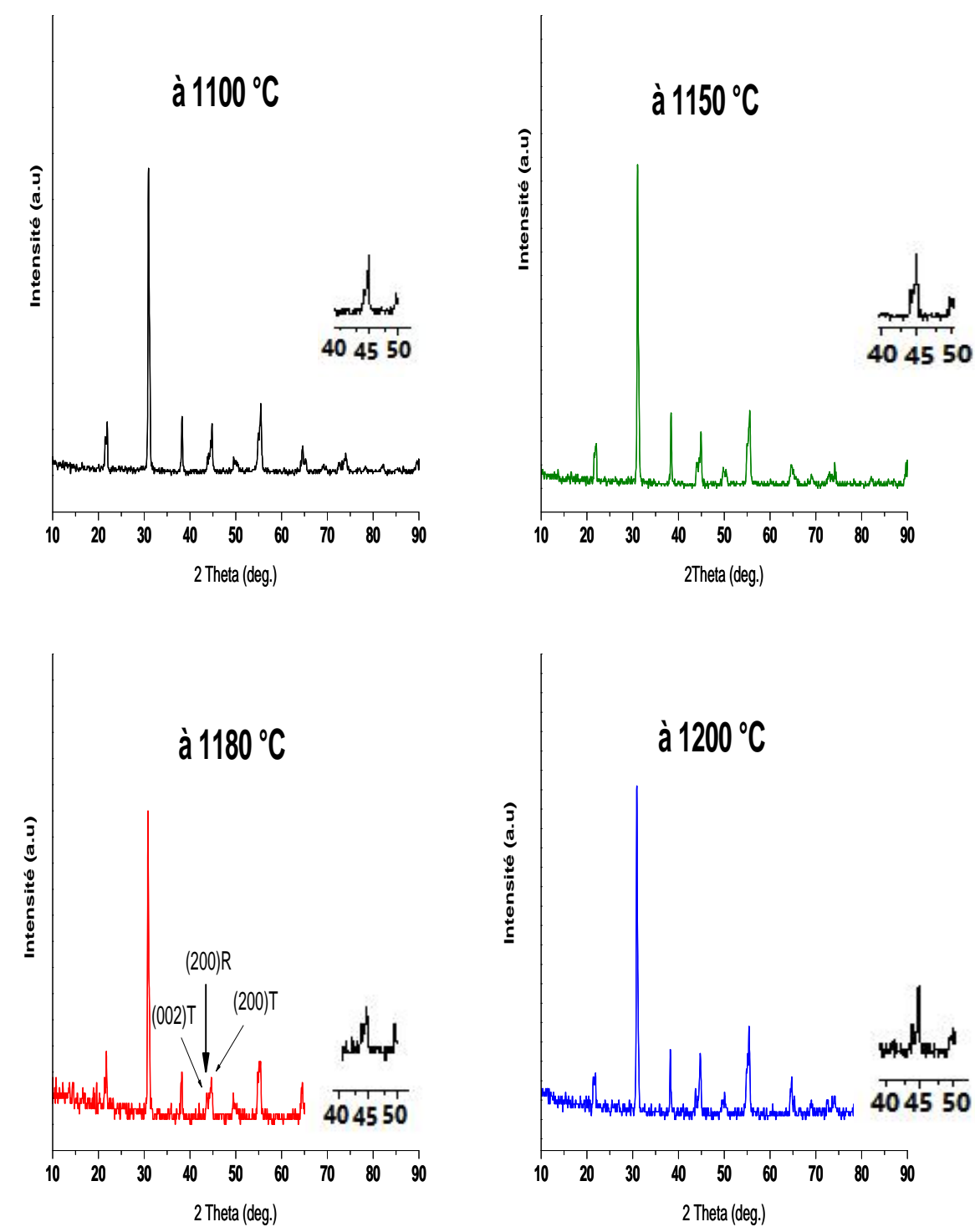

Fig.2. Diagramme de DRX de la poudre PZT-BCZTIS frittée à : $1100^{\circ} \mathrm{C}, 1150^{\circ} \mathrm{C}, 1180^{\circ} \mathrm{C}$ et $1200{ }^{\circ} \mathrm{C}$.

On constate sur la figure. 2 que les différents échantillons présentent une structure biphasique $(\mathrm{T}+\mathrm{R})$ par la présence simultanée d'un doublé de raies $(200)_{\mathrm{T}},(002)_{\mathrm{T}}$ et d'un raie simple $(200)_{\mathrm{R}}$ , indiquant la coexistence des deux phase ; tetragonale et rhomboédrique respectivement [1012] .

Pour tous les échantillons frittés. Ce triplet de raie présent à $2 \theta: 43-47$ est mieux mis en évidence sur les difractogrammes des échantillons frittés à $1180{ }^{\circ} \mathrm{C}$ et $1200{ }^{\circ} \mathrm{C}$ suite à 
l'éclatement des rais des deux phases $\mathrm{T}+\mathrm{R}$ indiquant la stabilité de ces phases à ces températures de frittage.

La densité de la composition PZT-BCZTIS est étudiée en fonction de la température de frittage.

Cette étude est nécessaire afin d'optimiser la température de frittage maximale, car la qualité du matériau augmente avec l'augmentation de la densité et celle-ci augmente avec l'augmentation de la température de frittage [13].

Les mesures de la densité sont effectuées sur des échantillons en pastilles frittés dans la gamme de température $1100-1200{ }^{\circ} \mathrm{C}$. Rappelant que, pour chaque température la densité expérimentale est calculée à partir des mesures géométriques de disque $(\Phi, \mathrm{e})$. Les résultats de ces mesures sont présentés sur la figure 3.

D'après cette figure, on remarque que la densité augmente en augmentant la température de frittage jusqu'à atteindre une valeur maximale à $1180{ }^{\circ} \mathrm{C}$ qui est de l'ordre $7,6477 \mathrm{~g} / \mathrm{cm}^{3}$ puis elle diminue. Cette température est donc, la température de frittage optimale pour la céramique PZT-BCZTIS.

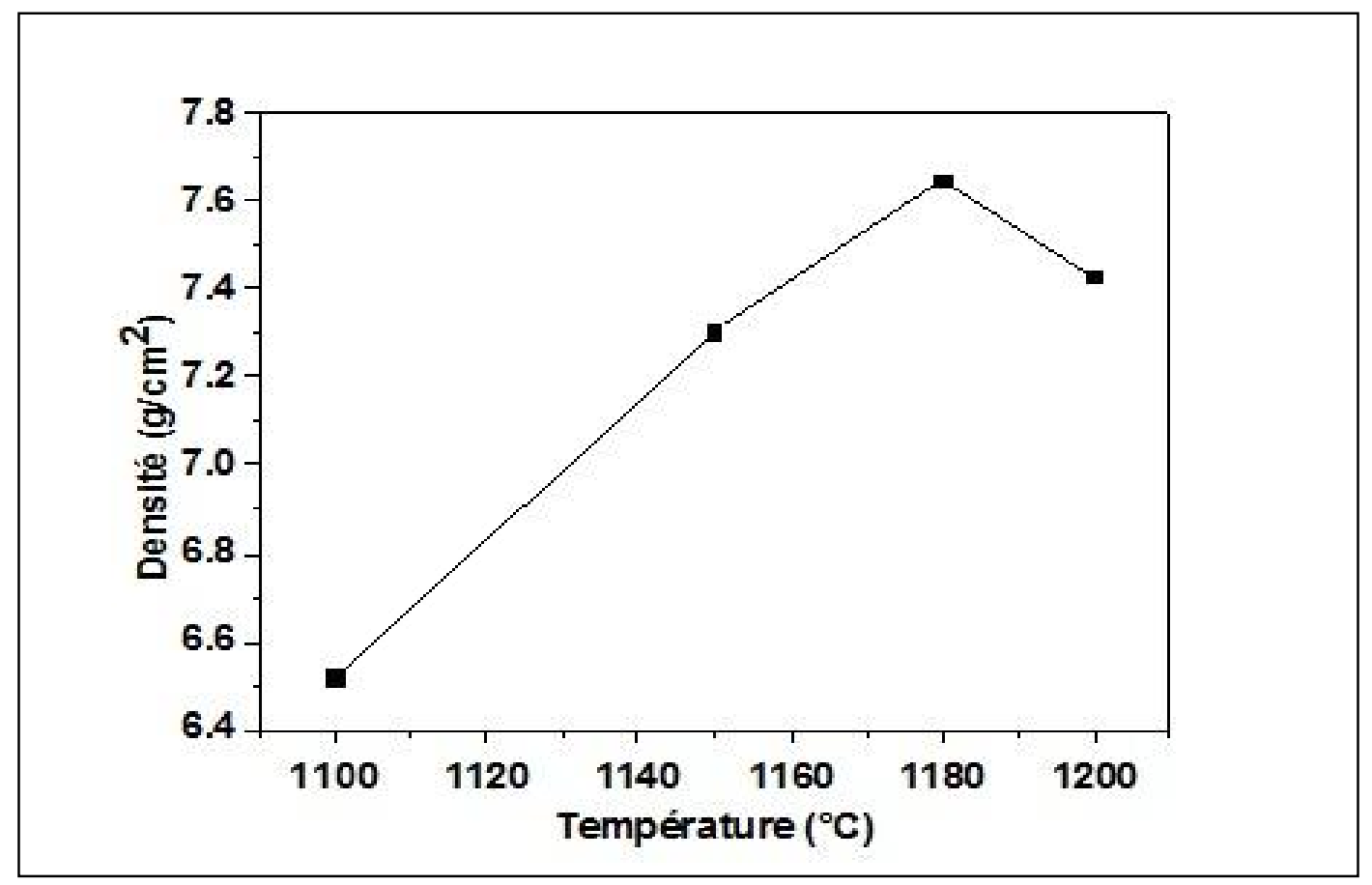

Fig.3. Évolution de la densité de PZT-BCZTIS en fonction de la température de frittage

L'augmentation de la densité implique une diminution du nombre et de dimension des pores, qui signifie que le volume de la maille diminue et par conséquent la structure devient plus compacte.

Il est important de signaler que la température de frittage optimal dépend de l'atmosphère de frittage. Alors la quantité de la poudre $\mathrm{PZrO}_{3}$, ajoutée pour minimiser la volatilisation de $\mathrm{PbO}$, 
a été contrôlée pour chaque frittage. Ainsi, la perte ou l'excès de masse a été contrôlée en pesant l'échantillon avant et après frittage. Notant qu'elle ne doit pas dépasser une valeur de $\mathrm{m} \leq 2 \%$. La température optimale de frittage correspond à l'équilibre évaporationdécondensation de $\mathrm{PbO}[14]$.

L'analyse par MEB apporte des informations sur la forme et la taille des grains après frittage des échantillons. Elle permet d'évaluer qualitativement la présence de la porosité et les phases secondaires. L'uniformité et l'homogénéité de notre céramique après chaque frittage est évaluée d'après les micrographes illustrés dans la figure 4.
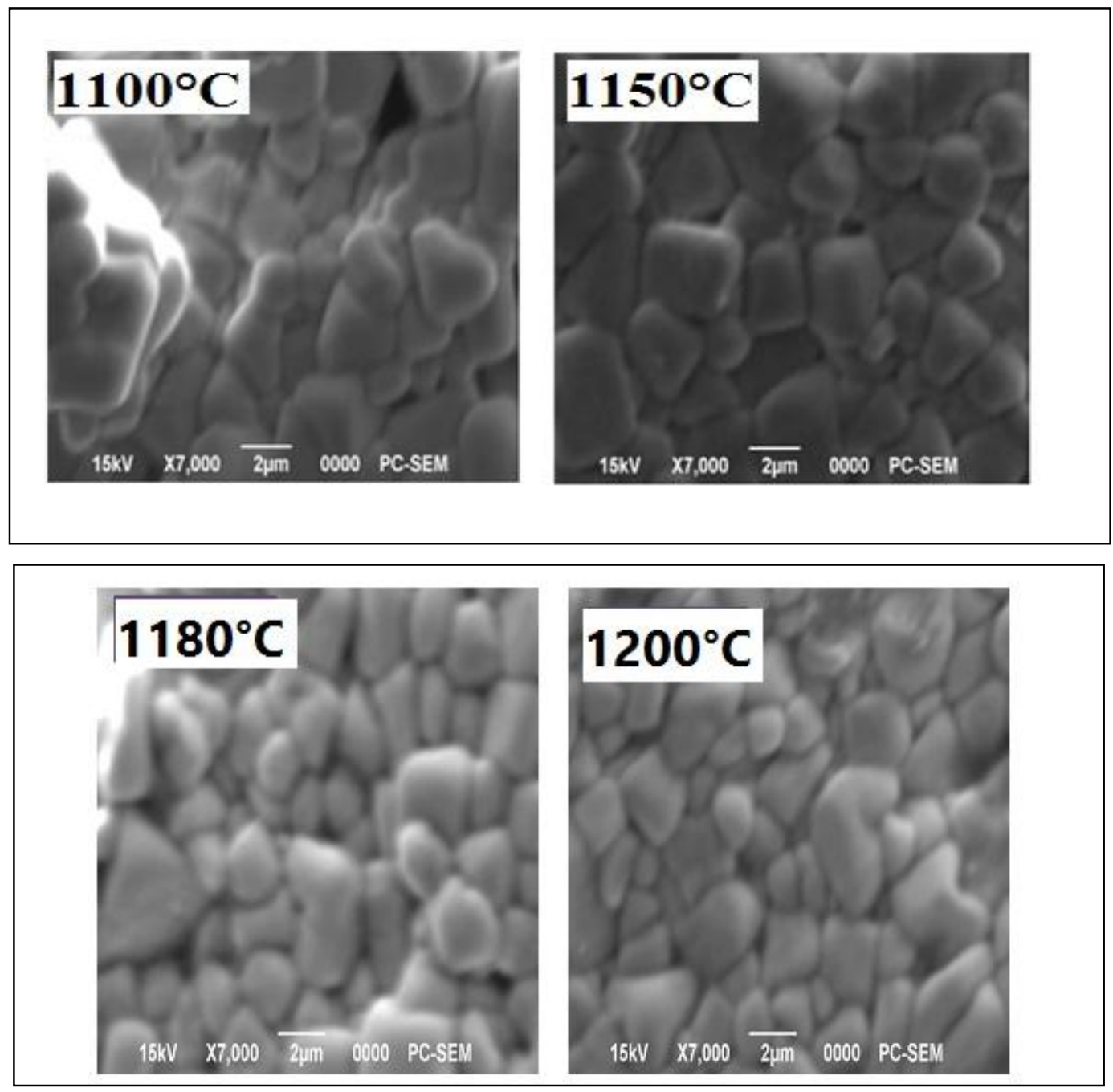

Fig.4. Micrographies de la céramique PZT-BCZTIS frittée

L'observation au MEB des poudres PZT-BCZTIS frittés à différentes températures a révélé une légère déférence dans la morphologie et la taille moyenne des grains. On observe un grossissement des grains au fur et à mesure que la température de frittage augmente, qui vient confirmer les résultats des observations de la densité. De même, l'échantillon fritté à $1180{ }^{\circ} \mathrm{C}$ 
présente une grande similitude morphologique, une surface plus dense et presque non poreuse constitue de grains aillons une taille moyenne de 2,634 $\mu \mathrm{m}$.

La figure 5 présente l'évolution de la constante diélectrique en fonction de la température pour l'ensemble des échantillons frittés à 1100, 1150, $1180^{\circ} \mathrm{C}$ à une fréquence de $1 \mathrm{KHz}$. Les courbes $\varepsilon_{\mathrm{r}}(\mathrm{T})$ présente la même allure, c'est-à-dire augmentation avec la température en passent par un maximum puis diminuent. Bien évidement ces courbes diffèrent selon la position et l'intensité de leur sommet. Ce dernier, dont la température correspond à celle de température de Curie $\mathrm{T}_{\mathrm{C}}$ est relier à la transition feroélectrique-paraélectrique. Aux hautes températures, les céramiques perdent tous leurs caractères ferroélectriques (phase de haute symétrie) ; ceci explique la décroissance de la constante diélectrique.

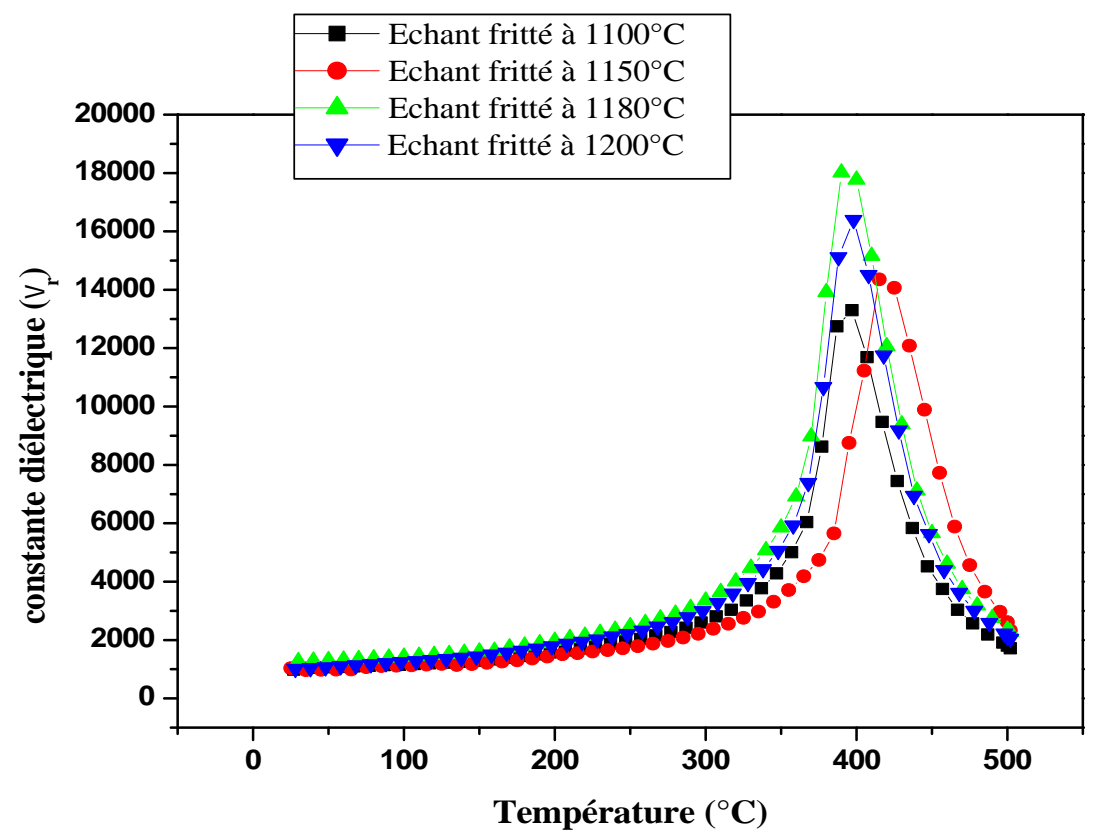

Fig.5. Evolution de la constante diélectrique en fonction de la température pour l'échantillon fritté à : $1100,1150,1180$ et $1200{ }^{\circ} \mathrm{C}$.

Notons que l'échantillon fritté à $1180{ }^{\circ} \mathrm{C}$ présente une valeur maximale de la constante diélectrique qui est de l'ordre 18018.

Lorsqu'un matériau ferroélectrique est sollicité électriquement ou mécaniquement il apparait au cours de la conversion de l'énergie des pertes diélectriques et mécaniques. La figure 6 représente la variation de l'angle des pertes en fonction de la température de frittage $\left(1100^{\circ} \mathrm{C}\right.$, 1150,1180 et $\left.1200^{\circ} \mathrm{C}\right)$. 


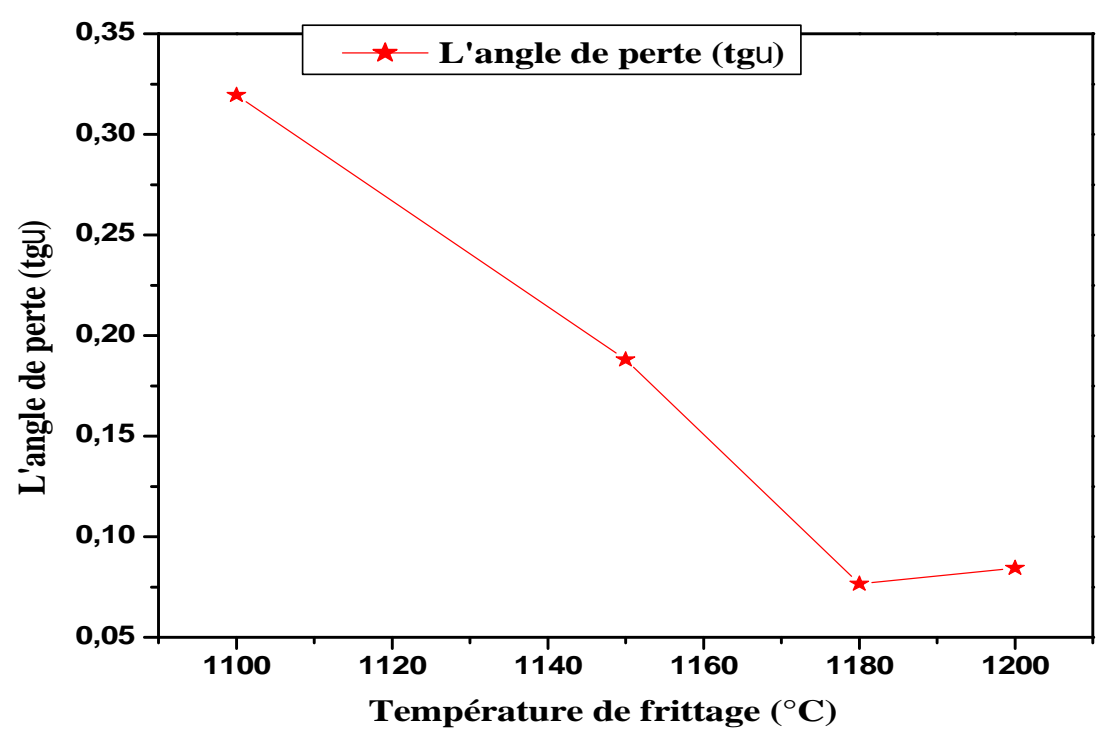

Fig.6. Variation du facteur de dissipation en fonction de la température de frittage.

Le facteur de dissipation décroît avec l'augmentation de la température de frittage jusqu'à atteindre des valeurs minimales de 0.07662 pour l'échantillon fritté à $1180{ }^{\circ} \mathrm{C}$. Ceci confirme une fois de plus que $1180{ }^{\circ} \mathrm{C}$ est la température optimale où la céramique est plus dense.

La mesure de la résistivité des échantillons dopés permet de mieux caractériser leur qualité électrique.

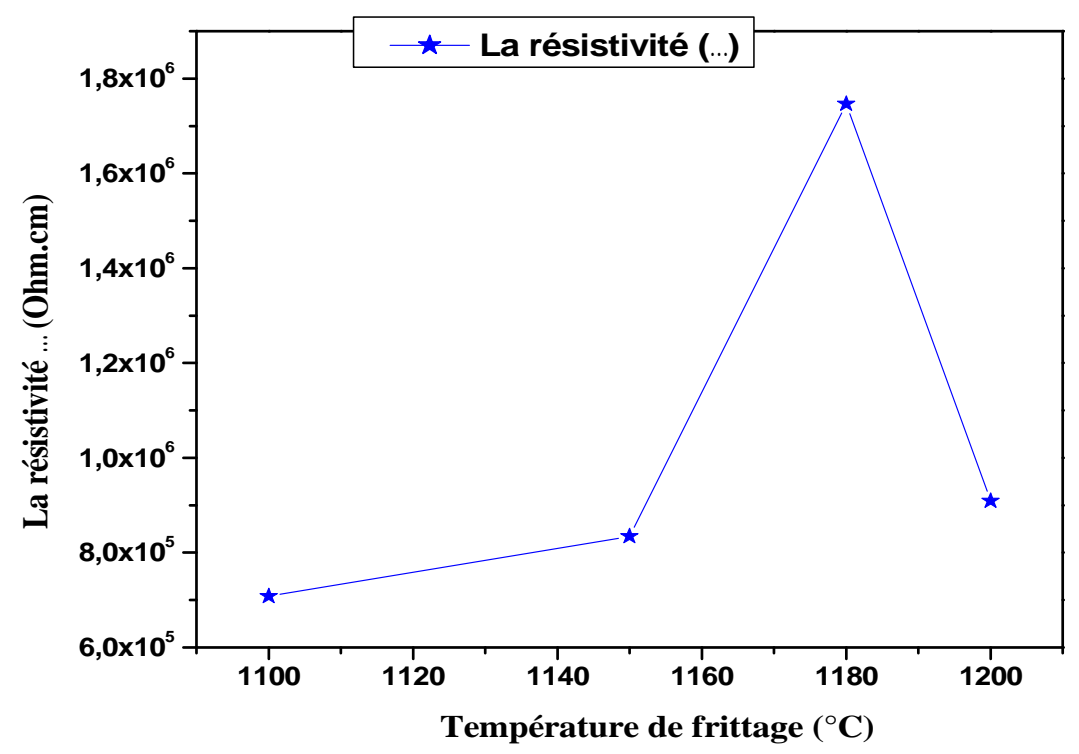

Fig.7. Variation de la résistivité en fonction de la température de frittage 
La figure 7 représente l'effet de la température de frittage sur la variation de la résistivité pour notre échantillon. Cette courbe montre une augmentation de la résistivité en fonction de la température de frittage de $1100{ }^{\circ} \mathrm{C}$ à $1150{ }^{\circ} \mathrm{C}$, jusqu'à une maximum valeur à $1180{ }^{\circ} \mathrm{C}$ qui est de l'ordre $\mathbf{1 . 7 4 6 4 9 * 1 0}^{6} \quad \mathbf{. c m}$, puis diminue à $1200^{\circ} \mathrm{C}$.

\section{CONCLUSION}

Dans cette étude l'objectif principal repose sur la synthèse et la caractérisation diélectrique du système quaternaire de formule générale :

$\mathrm{Pb}_{0.96} \mathrm{Ba}_{0.02} \mathrm{Ca}_{0.02}\left[\left(\mathrm{Zr}_{0.52} \mathrm{Ti}_{0.48}\right)_{0.94}\left(\mathrm{Zn}_{1 / 3} \mathrm{Ta}_{2 / 3}\right)_{0.03}\left(\mathrm{In}_{1 / 3} \mathrm{Sb}_{2 / 3}\right)_{0.03}\right] \mathrm{O}_{3}$ abrégé PZT-PBC-ZTIS. Un traitement thermique à différentes température $\left(1100,1150,1180\right.$ et $\left.1200{ }^{\circ} \mathrm{C}\right)$ est appliqué sur notre échantillon pour suivre et observer son comportement. Des mesures électriques ont été réalisées par des méthodes simples directes mais précises. L'analyse par diffraction des rayons $\mathrm{X}(\mathrm{DRX})$ a montré que la poudre après la calcination et le frittage est inclus dans la zone morphotropique (FMP) présentant deux phases quadratique et rhomboédrique. De point de vue morphologique, l'échantillon fritté à $1180^{\circ} \mathrm{C}$ est le plus dense $\left(\mathbf{d}=\mathbf{7 , 6 4 7 7} \mathbf{g} / \mathbf{c m}^{3}\right)$ donc cette température est la température optimale de frittage. La réponse diélectrique en fonction de la température de frittage des PZT-PBC-ZTIS a montré que l'échantillon fritté à $1180^{\circ} \mathrm{C}$ près de la FMP présente une forte permittivité diélectrique (18018.00) et un faible facteur de dissipation (0,07662). On peut conclure l'importance d'avoir des compositions denses et de structure biphasiques $(\mathrm{T}+\mathrm{R})$, pour avoir une forte réponse diélectrique.

\section{REFERENCES}

[1] JAFFE B., ROTH R.S., MARZULLO S., Journal of Research of the National Bureau of Standards, 1955, 55, 239-254.

[2] JAFFE B., SMOTRAKOW W. G., JAFF H, Piezoelectric ceramics. New York: Academic Press, 1971, pp.117.

[3] EREMKIN W., SMOTRAKOW W. G., FESENKO E. G., Sov. Solid State Phys, 1989, 31, p156-160.

[4] VIEHLAND D., Phys. Rev. B 52, 1995, 778-791.

[5] SINGH P. K., COCHRANE S., LIU W. TU., Appl. Phys. Lett. 66, 1995, 3683-3685.

[6] YONEDA Y., SAKAUE K., TERAUCHI H., Jpn. J. Appl. 2000, 39, 4839-4842. 
[7] BOUTARFAIA A, Etude de la transitions de phase de la céramique de type zirconatetitanate de plomb note $\mathrm{PZT}$ dans le système : $\mathrm{xPbZrO} 3-\mathrm{yPbTiO}_{3}-\mathrm{ZPb}\left(\mathrm{Fe}_{0.2}^{+3} \quad \mathrm{Ni}^{+2}{ }_{0.2} \mathrm{Sb}^{+5}{ }_{0.06}\right)$ $\mathrm{O}_{3}$, thèse de magister, Université de Constantine 1992.

[8] NECIRA Z, Étude structurale et physique des céramiques à base d'oxyde de type PZT, Thèse Magister, Université Biskra, 2005.

[9] ABBA M, Synthèse, caractérisation et étude des propriétés piézo-électriques des céramiques de type PZT, Thèse de Doctorat, Université de Biskra, 2013. [10] BOUTARFAIA A., BOUAOUD S. E., J. Ceram. Inter, 1996, 22, 281-286.

[11] BOUTARFAIA A., J. Ceram. Inter, 2001, 27, 91-97.

[12] ARI. GUR P., BENGUIGUI L., J. Phys. D: Appl. Phys, 1975, 8, 1856-1862.

[13] KELLY J., LEONARD M., TANTIGATE C., SAFARI A., J. Am. Ceram. Soc, 1997, 80, 957-964.

[14] OHTAKA O., VON DER MUHL R., RAVEZ J., J. Am. Ceram. Soc, 1995, 78, 805-808.

\section{How to cite this article:}

Abba M, Necira Z, Abdessalem N, Meklid A, Menasra H, Boutarfaia A. Elaboration and dielectric characterization of a doped ferroelectric material TYPE PZT. J. Fundam. Appl. Sci., 2013, 5(2), 210-219 